\title{
Multispectral microimager for astrobiology
}

R. Glenn Sellar, Jack D. Farmer, Andrew Kieta, Julie Huang

R. Glenn Sellar, Jack D. Farmer, Andrew Kieta, Julie Huang, "Multispectral microimager for astrobiology," Proc. SPIE 6309, Instruments, Methods, and Missions for Astrobiology IX, 63090E (14 September 2006); doi:

$10.1117 / 12.682272$

SPIE. Event: SPIE Optics + Photonics, 2006, San Diego, California, United States 


\title{
Multispectral Microimager for Astrobiology
}

\author{
R. Glenn Sellar ${ }^{* a}$, Jack D. Farmer ${ }^{\mathrm{b}}$, Andrew Kieta ${ }^{\mathrm{c}}$, and Julie Huang ${ }^{\mathrm{d}}$ \\ ${ }^{\mathrm{a}} \mathrm{Jet}$ Propulsion Laboratory, California Institute of Technology, ${ }^{\mathrm{b}}$ Arizona State University, ${ }^{\mathrm{c}} \mathrm{Mt}$. San Antonio College, \\ ${ }^{\mathrm{d} C a l i f o r n i a}$ Institute of Technology
}

\begin{abstract}
A primary goal of the astrobiology program is the search for fossil records. The astrobiology exploration strategy calls for the location and return of samples indicative of environments conducive to life, and that best capture and preserve biomarkers. Successfully returning samples from environments conducive to life requires two primary capabilities: (1) in situ mapping of the mineralogy in order to determine whether the desired minerals are present; and (2) nondestructive screening of samples for additional in-situ testing and/or selection for return to laboratories for more in-depth examination. Two of the most powerful identification techniques are micro-imaging and visible/infrared spectroscopy. The design and test results are presented from a compact rugged instrument that combines micro-imaging and spectroscopic capability to provide in-situ analysis, mapping, and sample screening capabilities.

Accurate reflectance spectra should be a measure of reflectance as a function of wavelength only. Other compact multispectral microimagers use separate LEDs (light-emitting diodes) for each wavelength and therefore vary the angles of illumination when changing wavelengths. When observing a specularly-reflecting sample, this produces grossly inaccurate spectra due to the variation in the angle of illumination. An advanced design and test results are presented for a multispectral microimager which demonstrates two key advances relative to previous LED-based microimagers: (i) acquisition of actual reflectance spectra in which the flux is a function of wavelength only, rather than a function of both wavelength and illumination geometry; and (ii) increase in the number of spectral bands to eight bands covering a spectral range of 468 to $975 \mathrm{~nm}$.
\end{abstract}

Keywords: Multispectral imager, microimager, astrobiology

\section{INTRODUCTION}

Perhaps the most basic tools for interpreting the origin of rocks in the field are the microscopic hand lens and rock hammer. A rock hammer is important in providing access to unweathered rock surfaces. However, a hand lens provides essential microtextural and compositional (mineralogical) information needed to discriminate between basic rock types and genetic processes. A systematic approach for analyzing the geology of a field site typically requires: 1) selecting a target rock based on precursor remote observations, 2) documenting bedding styles, mesoscale lithological structures and rock fabrics, 3) determining the bedding attitudes (strike and dip of beds) and any uncomformable bedding surfaces visible in outcrop, 4) preparing a fresh rock surface, 5) comparing the microtexture of weathered and unweathered rock surfaces, at magnifications of 10-30x,6) identifying the mineralogy of the rock based on the physical properties of any visible mineral phases present, 7) classifying the rock based on the combination of microtextural and mineralogical information. Such observations provide a framework for initial assessments of the paleoenvironmental context and history of rocks in the field, as well as the refinement of working hypotheses that drive decisions about what to do next. In addition, steps 4 and 5 can also lead to the detection of fossil biosignatures.

\footnotetext{
* glenn.sellar@jpl.nasa.gov; phone 818-354-4229; Jet Propulsion Laboratory, 4800 Oak Grove Drive, MS 170-B1, Pasadena, CA 91109
}

Instruments, Methods, and Missions for Astrobiology IX, edited by Richard B. Hoover, Gilbert V. Levin, Alexei Yu. Rozanov, Proc. of SPIE Vol. 6309, 63090E, (2006) · 0277-786X/06/\$15 - doi: 10.1117/12.682272 
Microtextural analysis of rocks using a hand lens, when combined with a knowledge of mineralogy, provides a powerful combination for assessing the origin of a rock. Armed with such information, the trained field geologist is often a position to immediately assign a rock to one of three basic petrogenetic categories (igneous, sedimentary or metamorphic) and to begin to interpret past geological processes based on the interplay of textural and compositional information. In addition, comparisons of weathered and unweathered surfaces can reveal important information about the geologic processes currently active at a site.

While experience suggests that much of the basic information needed to interpret the paleoenvironmental context of a rock can be obtained in the field using the simple field methods described above, for precise mineral identifications, quantitative mineral abundances and identification of minor mineral phases present requires more sophisticated lab analyses. In standard geological applications, lab work usually focuses on the preparation of samples for light (transmitted, reflected and luminescence) and electron (reflected and transmission) microscopy, with supporting geochemical information being provided by such methods as SIMS, or the electron microprobe. While these are common capabilities of most geology labs, their counterparts for in situ analyses are quite limited by the need to be small, lightweight, and rugged. Contact instruments that can analyze the composition and texture of rocks and soils have a clear advantage over other methods, in requiring little, if any, sample preparation.

\section{COMPACT MICROIMAGING SPECTROMETER}

An initial, 8-band version of the Compact Micro-Imaging Spectrometer (CMIS) has been designed, integrated and tested, and is shown in Figure 1. As an imaging spectrometer, CMIS provides a reflectance spectrum for every pixel in its field-of-view (FOV). The design employs multi-wavelength light emitting diode (LED) illumination to provide these reflectance spectral data cubes, with no moving parts. Performance parameters are summarized in Table 1. A custom micro-imaging lens for 400 to $1700 \mathrm{~nm}$ was designed and fabricated for this instrument, though the focal plane array currently in use is sensitive only from 400 to $1000 \mathrm{~nm}$. This lens was designed with a slow focal ratio (F/10) to provide a $\sim 2 \mathrm{~mm}$ depth of field suitable for imaging unprepared surfaces. The digital data is converted to reflectance using a 2-point linear calibration.

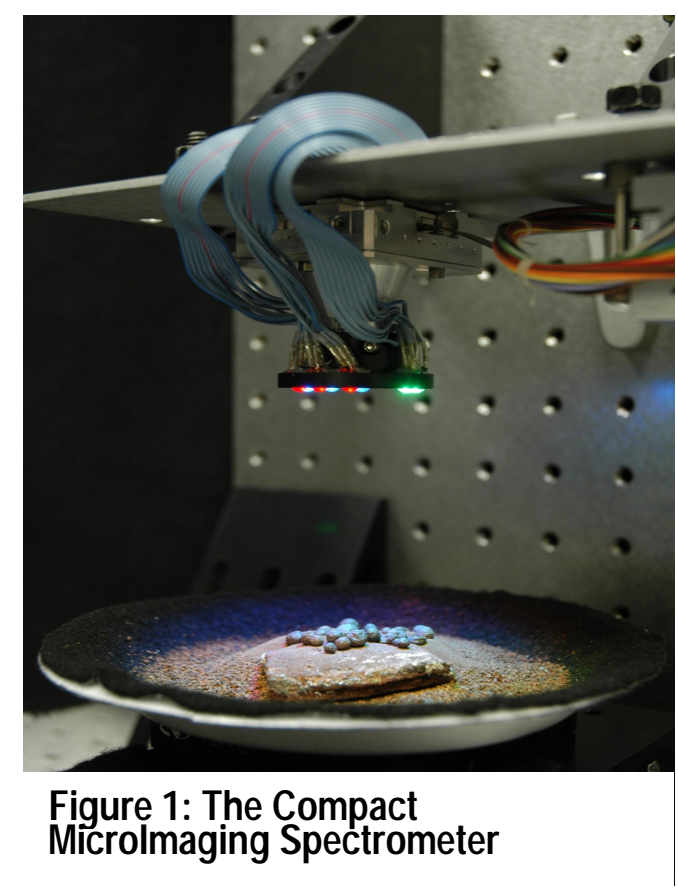

Table 1: Performance of the 8-band CMIS

Spatial Samples: $\quad 1024 \times 1024$

Spatial Resolution: $\quad 30 \mu \mathrm{m}$

Field-of-View: $\quad 30 \times 30 \mathrm{~mm}$

Digitization: 12-bits

Spectral band centers: $468 \mathrm{~nm}$

$525 \mathrm{~nm}$

$660 \mathrm{~nm}$

$735 \mathrm{~nm}$

$805 \mathrm{~nm}$

$850 \mathrm{~nm}$

$940 \mathrm{~nm}$

$975 \mathrm{~nm}$ 
Accurate reflectance spectra should be a measure of reflectance as a function of wavelength only, but instruments that use separate LEDs (light-emitting diodes) for each wavelength ${ }^{12}$ will also change the angles of illumination when changing wavelengths. This approach produces grossly inaccurate spectra as illustrated in Figure $2 \mathrm{c}$. The gray hematite sample shown in Figure 2c does not actually preferentially reflect the red, green, or blue wavelengths exhibited in this image; these features are spurious artifacts caused by the variation in the angle of illumination (heterotropic illumination as illustrated in Figure 2a). In contrast, the CMIS approach uses multi-wavelength LEDs, allowing the instrument to maintain the same angles of illumination for every wavelength (isotropic illumination as illustrated in Figure $2 b$ ). This advance eliminates the grossly inaccurate spectra produced by the prior approach, as illustrated in Figure 2d.

The single-wavelength approach also exhibits instrumental artifacts when the sample has crevices or shadowed spaces between particles. In this case, portions of the object are in shadow relative to some but not all of the LEDs. For instruments that use separate LEDs for each wavelength, this effect results in grossly inaccurate spectra for these partially-shadowed areas of the object. This latter effect occurs with diffuse as well as specular objects (if they have deep pits, crevices, or partially-shadowed spaces between particles).

\section{PRELIMINARY RESULTS}

Figure 3 compares the data obtained at a hand-lens scale with white-light illumination to that obtained by CMIS in three visible bands. The samples are hematite concretions from Snow Canyon, UT, on a background of palagonite particles. Figure 4 shows preliminary results from the 8-band version of the CMIS observing a set of mineralogical samples: an approximately-true color image comprised of three of the eight spectral bands and the reflectance spectra of selected sets of pixels. The ability to extract the spectra of any pixels or sets of pixels enables distinct spectra to be distinguished for any desired features or areas in the sample. The mean spectrum for the entire field of view is also shown, to illustrate the type of data that would be obtained by a non-imaging spectrometer.

\section{CONCLUSIONS}

These test results demonstrate the successful development and the capability of a rugged, lightweight, 8-band multispectral microimager with no moving parts. The use of multiwavelength LEDs in the CMIS design eliminates the instrumental artifacts exhibited by instruments that use separate LEDs for each wavelength. The CMIS approach show great promise for extension to larger numbers of spectral bands and extension of the spectral range further into the infrared.

\section{ACKNOWLEDGEMENTS}

The research described in this paper was carried out at the Jet Propulsion Laboratory, California Institute of Technology, under a contract with the National Aeronautics and Space Administration. This research was funded by the NASA's Astrobiology Science and Technology Instrumentation Development program under task number 409725.

\section{REFERENCES}

\footnotetext{
${ }^{1}$ N. Thomas, B. S. Lüthi, S. F. Hviid, H. U. Keller, W. J. Markiewicz, T. Blümchen, A. T. Basilevsky;1, P. H. Smith, R. Tanner, C. Oquest, R. Reynolds, J.-L. Josset, S. Beauvivre, B. Hofmann, P. Rüffer , and C. T. Pillinger, "The microscope for Beagle 2," Planetary and Space Science 52, 853 - 866 (2004).

${ }^{2}$ M. H. Hecht, T. P. Meloy, and J. R. Marshall, "The Mars Environmental Compatibility Assessment (MECA)," Concepts and Approaches for Mars Exploration, 6186, (Lunar and Planetary Institute, Houston, TX, July 18-20, 2000).
} 
(a)

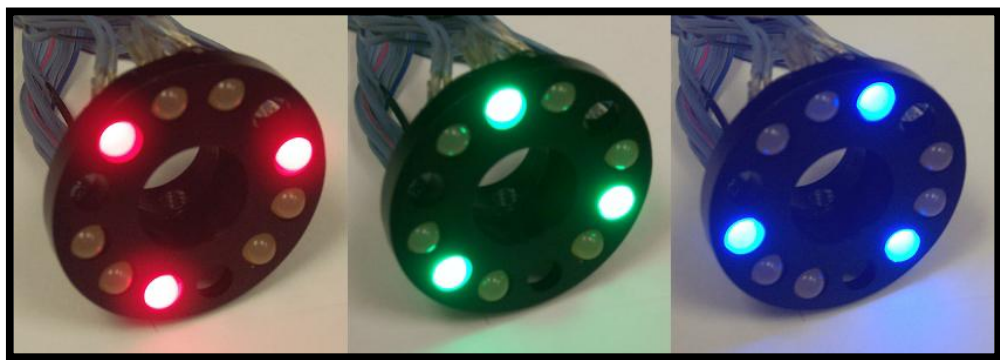

(b)

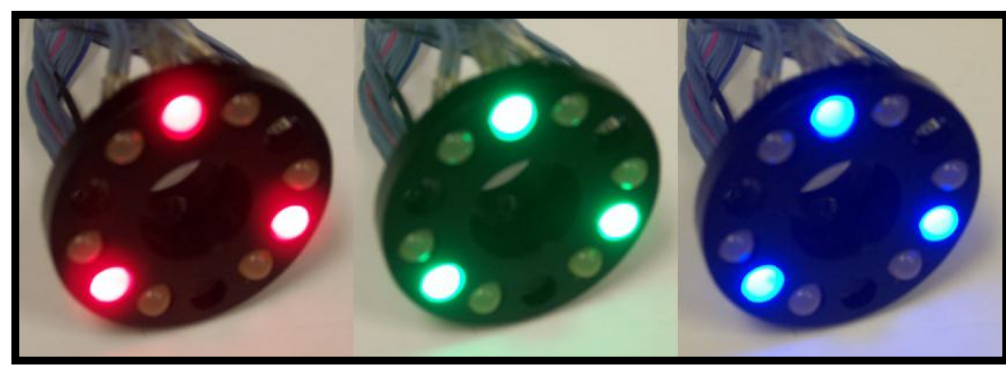

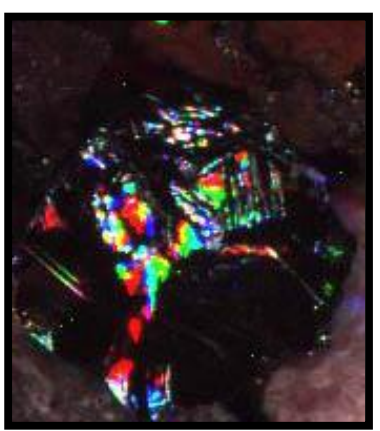

(c)

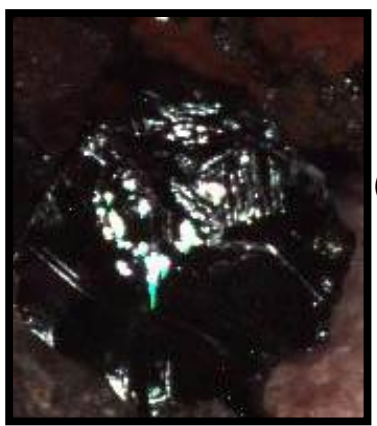

(d)

Figure 2: Demonstration of the effect of using separate single-wavelength LEDs versus multiwavelength LEDs; (a) heterotropic illumination sequence as used by instruments with singlewavelength LEDs in which each wavelength illuminates the object from different set of angles; (b) isotropic illumination sequence using multi-wavelength LEDs in which the illumination angles do not vary; (c) composite image of gray hematite acquired with heterotropic illumination; (d) composite image of the same sample acquired using isotropic illumination

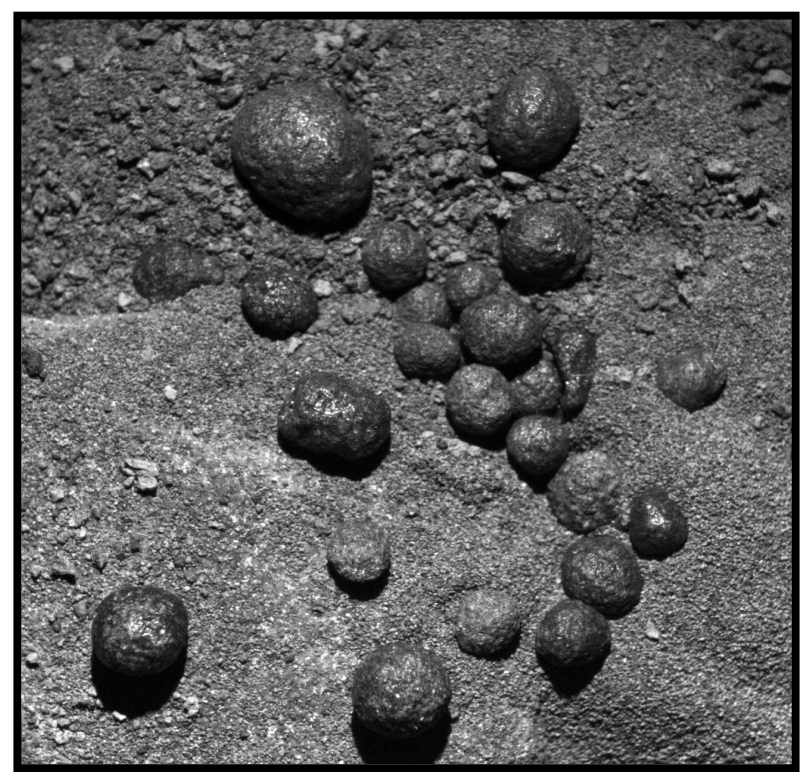

(a)

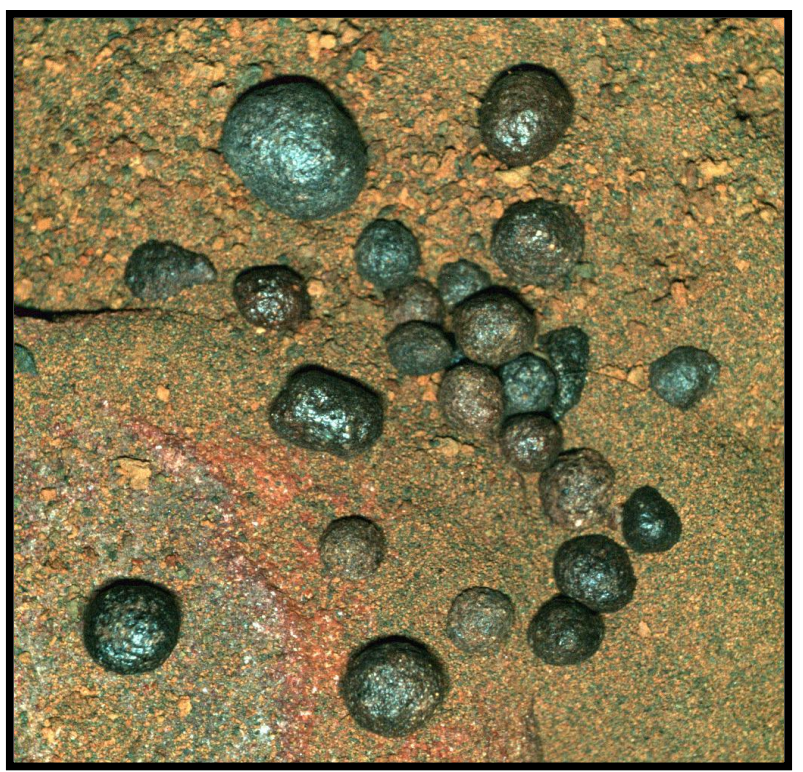

(b)

Figure 3: Hematite concretions from Snow Canyon, Utah on a background of palagonite; (a) Panchromatic image acquired with white-light illumination compared to; (b) 3-band multispectral image acquired with the CMIS. The field-of-view is $\sim 30 \mathrm{~mm}$ square with a resolution of $30 \mu \mathrm{m}$. Band centers for the 3-band image were $468 \mathrm{~nm}$ (blue), $525 \mathrm{~nm}$ (green), and $660 \mathrm{~nm}$ (red). 
Figure 4: Collection of geological samples: red hematite, gray hematite, goethite, olivine, andesite, rhodochrosite, and palagonite; (a) approximately-truecolor image composed of three of the eight bands acquired by the CMIS; (b) calibrated reflectance spectra of the sets of pixels indicated in the key (c). The field-ofview is $\sim 30 \mathrm{~mm}$ square with a. resolution of $30 \mu \mathrm{m}$.

(a)

(b)
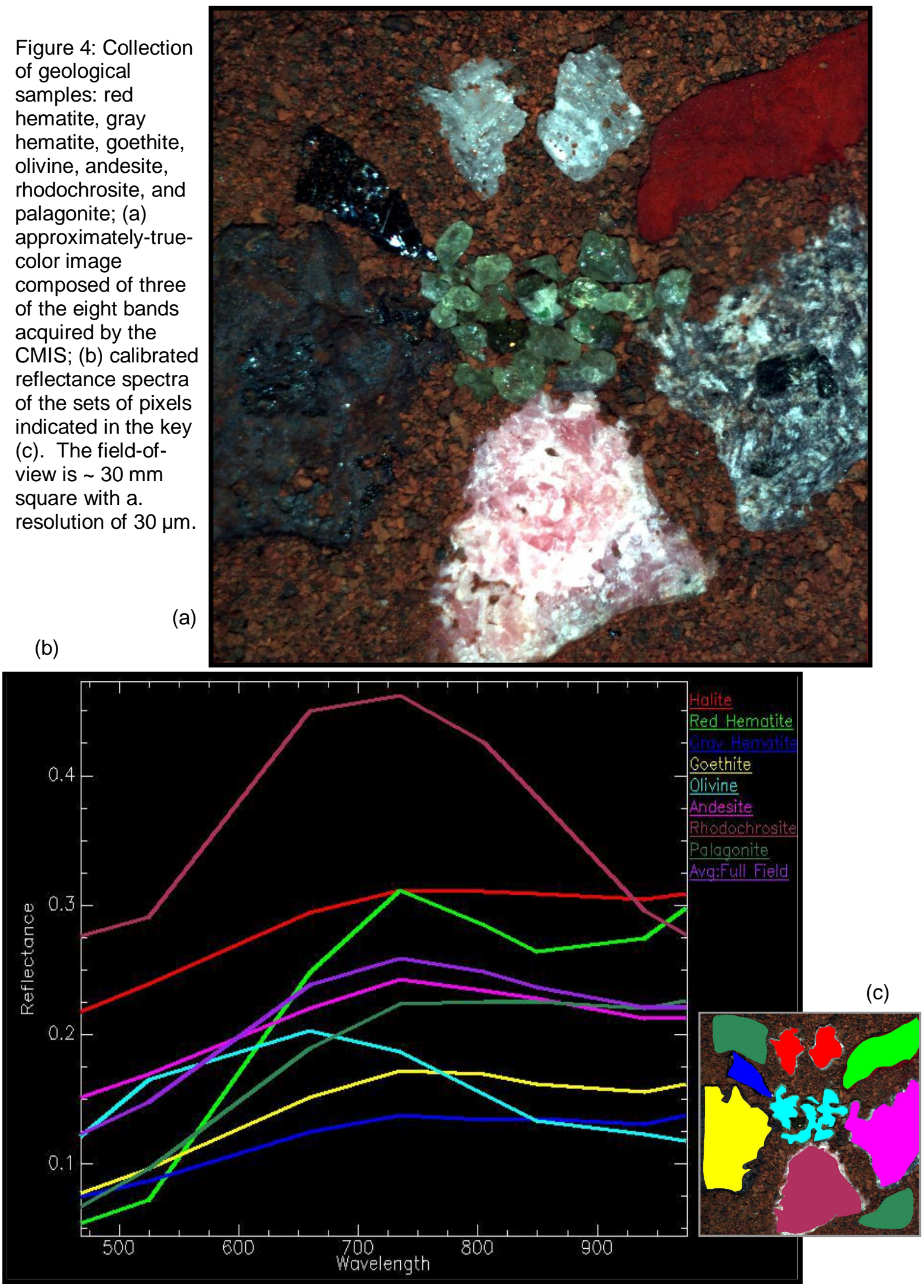